\title{
High Figure-of-Merit Laterally Fluorinated Biphenyltolane-Isothiocyanates
}

\author{
Sebastian Gauza \\ Yang Zhao \\ Tatiana Le Cor \\ Shin-Tson Wu \\ College of Optics and Photonics, University of Central Florida, \\ Orlando, FL, USA
}

\section{Anna Ziółek \\ Roman Dąbrowski}

Institute of Chemistry, Military University of Technology,

Warsaw, Poland

\section{Otilia Catanescu \\ Liang-Chy Chien}

Liquid Crystal Institute, Kent State University, Kent, OH, USA

\section{Chain-Shu Hsu}

Department of Applied Chemistry, National Chiao Tung University, Hsinchu, Taiwan

We have designed, synthesized, and evaluated the physical properties of some high birefringence $(\Delta n)$ isothiocyanato biphenyl-tolane liquid crystals. These compounds exhibit $\Delta n \sim 0.48-0.52$ at room temperature and wavelength $\lambda=633 \mathrm{~nm}$. Laterally substituted short alkyl chains and fluorine atom eliminate smectic phase and lower the melting temperature. The moderate melting temperature and very high clearing temperature make those compounds attractive for eutectic mixture formulation. A eutectic mixture based on those compounds was formulated and its physical properties evaluated.

This work is supported by DARPA Bio-Optics Synthetic Systems program under Contract No. W911NF04C0048, and NATO Programme Security Through Science, Collaborative Linkage Grant No. CBP.EAP.CLG 981323.

Address correspondence to Shin-Tson Wu, College of Optics and Photonics, University of Central Florida, Orlando, FL 32816, USA. E-mail: swu@mail.ucf.edu 
Keywords: eutectic mixtures; high birefringence; isothiocyanato bistolanes; liquid crystals

\section{INTRODUCTION}

High birefringence $(\Delta \mathrm{n})$ liquid crystals (LCs) support a wide field of different applications such as flat panel display, telecommunication, and tunable-focus lens. For these devices, high $\Delta \mathrm{n}$ improves response time through cell gap (d) reduction. This advantage is especially important for laser beam steering [1] and variable optical attenuator [2] as these devices are normally operated in the near infrared region $(\lambda=1.55 \mu \mathrm{m})$. In the long wavelength region, the $\mathrm{LC} \mathrm{d} \Delta \mathrm{n}$ should increase in order to achieve the required phase change, which depends on the specific LC alignment. High birefringence helps to reduce the cell gap so that the fast response time can still be maintained.

To achieve high birefringence, linearly conjugated molecules are the preferred candidates. Conjugation length can be extended by multiple bonds or unsaturated rings in the rigid core. Three problems associated with highly conjugated LC compounds are high melting temperature, increased viscosity and reduced UV stability. To overcome high melting temperature, we could form eutectic mixtures. The increased viscosity is inherent to all the highly conjugated compounds. However, isothiocyanato (NCS) compounds are less viscous than the cyano (CN) ones except that the NCS compounds tend to exhibit smectic phases [3]. To avoid UV instability, these high birefringence compounds are more suitable for infrared applications.

Several high birefringence molecular structures, such as diphenyldiacetylene [4,5], bistolane [6,7], naphthalene tolanes [8], and thiophenyl-diacetylene [9-11] have been studied. Recently some of biphenyl-tolane compounds were synthesized and their properties were investigated [12-14]. The birefringence of these compounds is in the 0.4-0.6 range. The diacetylene compounds do not have adequate UV and thermal stabilities [15] so that their application is limited.

In this article, we present some isothiocyanato biphenyl-tolane compounds with extrapolated birefringence $\sim 0.48-0.52$ at $\lambda=633 \mathrm{~nm}$ and $\mathrm{T}=23^{\circ} \mathrm{C}$. To lower melting temperature, we tried different lateral substitutions, such as alkyl and fluoro groups.

\section{EXPERIMENTAL}

Several measurement techniques were used to measure the physical properties of the single compounds and mixtures. A Differential 
Scanning Calorimetry (DSC, TA Instrument Model Q-100) was used to determine the phase transition temperatures. Results were obtained from $3-6 \mathrm{mg}$ samples in the heating and cooling cycles with rate $2{ }^{\circ} \mathrm{C} / \mathrm{min}$. The electro-optic properties of the LC compounds and mixtures were measured using an 5 and $8 \mu \mathrm{m}$ homogenous cells with ITO (indium-tin-oxide) electrodes coated in the inner sides of the glass substrates. A thin polyimide layer was overcoated on ITO and buffed in anti-parallel directions to produce a small pretilt angle $\left(\sim 2^{\circ}\right)$. A linearly polarized He-Ne laser with $\lambda=633 \mathrm{~nm}$ was used as the light sources for the electro-optic measurements. Experimental setup and technique are detailed in [16].

The phase retardation $(\delta)$ of the homogeneous cells was measured with the LabVIEW system. The LC birefringence $(\Delta n)$ at wavelength $\lambda$ and temperature T was then obtained by from the following Eq. [16]:

$$
\delta(V, T, \lambda)=\frac{2 \pi d \Delta n(V, T, \lambda)}{\lambda}
$$

\section{RESULTS AND DISCUSSIONS}

\subsection{Single Compounds}

Table 1 lists the molecular structures and phase transition temperatures of the five isothiocyanato biphenyl-tolane LCs investigated. Even with lateral substitution(s), the melting temperatures of these compounds are still relatively high. The mono-fluoro substituted compounds 1 and 2 exhibit a wide nematic range, but their melting temperatures are quite high $\left(\sim 146^{\circ} \mathrm{C}\right)$. Therefore, their solubility in eutectic mixtures would be limited to less than $5 \%$. Compound 5 , the biphenyl-tolane laterally substituted by a methyl group has a slightly lower melting point $\left(137^{\circ} \mathrm{C}\right)$, but there is a smectic phase stable up to $151.9^{\circ} \mathrm{C}$ before nematic phase appears. Moreover, the lateral methyl group would increase the rotational viscosity. Compound 3 has difluoro substitutions in the $(3,5)$ positions of the phenyl ring with the -NCS terminal group. These difluoro groups significantly lower the melting point to $65.3^{\circ} \mathrm{C}$, although a smectic phase exists during cooling. Compound 4 has another fluoro substitution in the second position of the middle phenyl ring. This fluoro substitution completely suppresses the smectic phase, but its melting point remains at $66.7^{\circ} \mathrm{C}$. For practical applications, the lateral substitution will increase viscosity and lower the birefringence. As a result, its overall figure-of-merit is reduced quite noticeably. 
TABLE 1 Molecular Structures and Phase Transition Temperatures of Five Biphenyl-Tolane Isothiocyanates

\begin{tabular}{lll}
\hline No & Structure & $\begin{array}{l}\text { Phase transition } \\
\text { temperature }\left({ }^{\circ} \mathrm{C}\right)\end{array}$ \\
\hline
\end{tabular}

1<smiles>CCc1ccc(-c2ccc(C#Cc3ccc(N(C)S)c(F)c3)cc2)cc1</smiles>

2

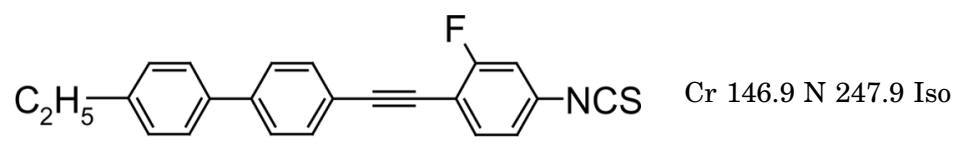

3

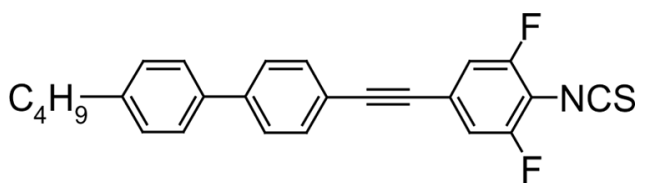

Cr 65.3 (S 50.1) N 212 Iso

4<smiles>CCCc1ccc(-c2ccc(C#Cc3cc(F)c(N=[Si])c(F)c3)cc2F)cc1</smiles>

Cr 66.7 N 172.5 Iso

5<smiles>CCc1ccc(-c2ccc(C#Cc3ccc(N(C)C)c(C)c3)cc2)cc1</smiles>

Most of device applications are performed at room temperature $\left(\sim 23^{\circ} \mathrm{C}\right)$. Thus, we would like to know the physical properties of these high melting compounds at room temperature. Two methods are commonly employed to extrapolate the electro-optical properties of these single compounds at room temperature: 1) guest-host system, and 2) fitting extrapolation from elevated temperature results. In the guest-host system, about $10 \mathrm{wt} \%$ of the guest compound to be investigated is dissolved in a room temperature LC host mixture. By knowing the properties of the host mixture, the properties of the guest 
compound at room temperature can be estimated. In the second method, we measure the properties of the compound at several temperatures in its nematic phase and then fit these data with theories, and finally extrapolate the results to room temperature. In this study, we focused on the birefringence $(\Delta \mathrm{n})$, visco-elastic coefficient $\left(\gamma_{1} / \mathrm{K}_{11}\right)$ and Figure-of Merit $\left(F_{O} M=K_{11} \Delta n^{2} / \gamma_{1}\right)$. For example, the birefringence of an LC can be described bt the following equation:

$$
\Delta n=\Delta n_{o}\left(1-T / T_{c}\right)^{\beta},
$$

where $\Delta \mathrm{n}_{\mathrm{o}}$ and $\beta$ are fitting parameters: $\Delta n_{\mathrm{o}}$ being the birefringence at $\mathrm{T}=0$ and $\beta$ is a material constant, and $T_{c}$ is the clearing temperature of the LC. By fitting experimental data with Eq. (2), we can obtain $\Delta n_{\mathrm{o}}$ and $\beta$. Once these two parameters are determined, the birefringence of the $\mathrm{LC}$ at room temperature can be extrapolated. In this study, we use the second method.

In experiment, we measured the temperature-dependent birefringence and visco-elastic coefficient and then calculated the FoM of the five biphenyl-tolane isothiocyanates listed in Table 1. Results are shown in Figures 1(a), 1(b), and 1(c), respectively. With no surprise, both single fluorinated biphenyl-tolane isothiocyanates have the highest birefringence among the five compounds investigated. Two reasons stand for this when we consider rod-like rigid compounds. More linear and fewer lateral substitution the structure is, the higher birefringence it shows. The second reason is their higher $T_{c}$. From Eq. (2), a higher $T_{c}$ is helpful to enhance birefringence, provided the molecular structure is similar.

The extrapolated $\Delta \mathrm{n}$ value at $25^{\circ} \mathrm{C}$ is 0.590 and 0.562 for compounds 1 and 2, respectively. Compound 2 has fluorine substitution in the 2nd position of the phenyl ring with NCS terminal group. Its clearing temperature is about 7 degrees lower than that of compound 1 in which the fluoro substitution is at the 3rd position of the phenyl ring. Another factor affecting birefringence is molecular packing. By comparing the simulated electrostatic charges on the surface of the molecules, the fluorine in the 2 nd position has higher negative charge than that in the 3 rd position. This suggests that the fluorine in the 2nd position is likely to drag out more $\pi$-electron clouds from the conjugated core than if the fluorine is substituted in the 3rd position of the same phenyl ring.

The substitution of the third lateral fluorine, as shown in compound 4, further decreases the clearing point so that its birefringence is lowered. There is no significant difference in birefringence between compounds 3 and 5 which has a methyl group substituted 


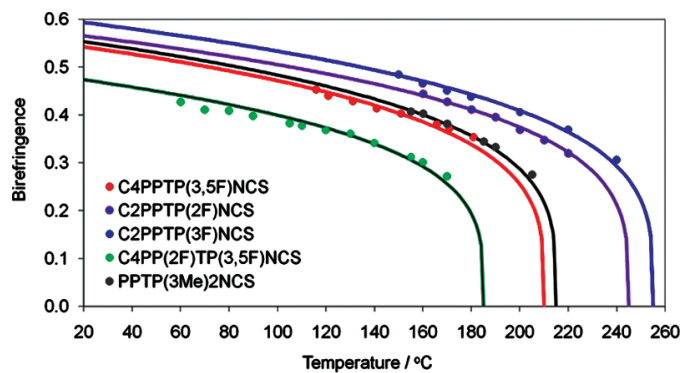

(a)

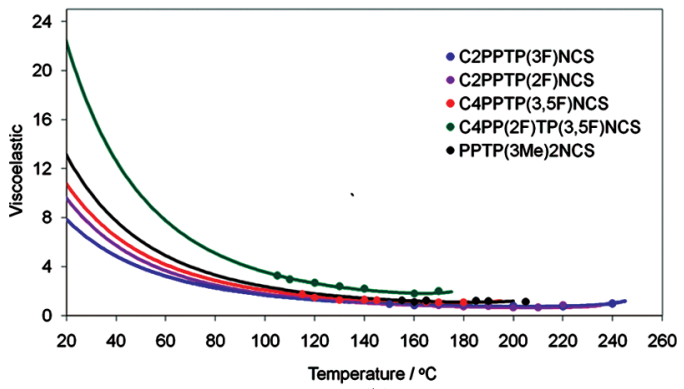

(b)

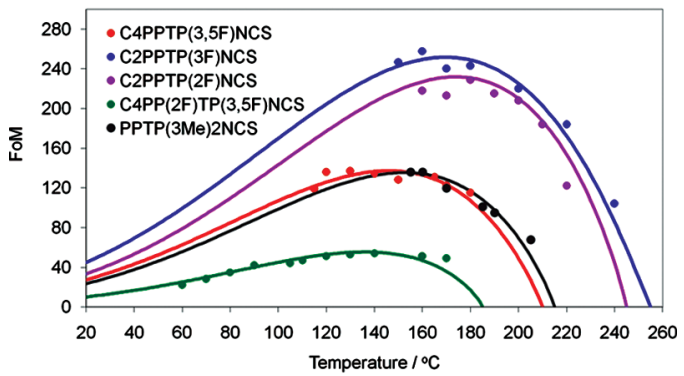

(c)

FIGURE 1 Temperature dependent (a) birefringence, (b) visco-elastic coefficient, and (c) figure-of-merit of the five single biphenyl-tolane compounds.

in 3rd position of the phenyl ring with the -NCS terminal group. The extrapolated birefringence for compounds 3 and 5 at $25^{\circ} \mathrm{C}$ is 0.539 and 0.550 , respectively. As shown in Table 1, the fluorinated compounds have better mesomorphic properties than the one with -Me group in lateral position. Triple fluorination does not lower melting point too noticeably for the investigated rigid core. It effectively destabilizes smectic phase but also decreases the clearing temperatures. 
The extrapolated birefringence for compound 5 to $25^{\circ} \mathrm{C}$ is 0.470 , which is the lowest among the five biphenyl-tolanes investigated.

Through free relaxation time measurement of a homogeneous cell, we are able to calculate the visco-elastic coefficient of the LC compounds. Results are depicted in Figure 1(b). Along with our prediction the compounds with fewer lateral substitutions should exhibit lower visco-elastic coefficient. From Figure 1(b), the following sequence is observed: $4>5>2>3>1$ at room temperature. As the temperature increases, the visco-elastic coefficient declines quickly. As $\mathrm{T}>110^{\circ} \mathrm{C}$, the difference between the five compounds becomes relatively small. With the exception for the triple fluorinated compound 5 , all other investigated compounds maintain almost the same value of $\gamma_{1} / K_{11}$ with slightly lower value for single fluorinated structures (compounds 1 and 2). Our measurement shows that introducing fluorine into lateral position in the biphenyl part of discussed structure (compound 4) significantly increases the visco-elastic coefficient.

Figure 1(c) shows the temperature dependent FoM calculated from data presented in Figures 1(a) and (b). Compounds 1 and 2 have the highest FoM among the five compounds studied. Their FoM reaches $250 \mu \mathrm{m}^{2} / \mathrm{s}$ and $230 \mu \mathrm{m}^{2} / \mathrm{s}$ at the optimal temperature of $171^{\circ} \mathrm{C}$ and $177^{\circ} \mathrm{C}$, respectively for compounds 1 and 2 . On the contrary, the maximum FoM of compound 4 is $\sim 40 \mu \mathrm{m}^{2} / \mathrm{s}$, which is $\sim 6 \mathrm{X}$ smaller than those of compounds 1 and 2 .

It is not practical to operate any LC device at such a high elevated temperature. The desired operating temperature is room temperature. Thus, we extrapolate FoM to $\mathrm{T} \sim 25^{\circ} \mathrm{C}$. At low temperature, viscoelastic coefficient increases significantly and FoM decreases to $50 \mu \mathrm{m}^{2} / \mathrm{s}$ and $40 \mu \mathrm{m}^{2} / \mathrm{s}$, respectively, for compounds 1 and 2 . These extrapolated results indicate that the single fluorinated biphenyltolane isothiocyanates are attractive for those applications where high birefringence is preferred. The FoM of the difluoro and methyl substituted biphenyl-tolane are quite similar as their birefringence and visco-elastic coefficient value are close to each other. Triple fluorinated biphenyl-tolane (compound 4) has the lowest FoM among the five compounds studied. High viscosity is a severe drawback of this structure, otherwise, it shows pure nematic phase.

\subsection{Eutectic Mixtures}

It is commonly known that only several single compounds possess liquid crystalline properties at room temperature. Thus, eutectic mixtures are mostly in use. The purposes of our experiment are twofold: 1) to evaluate the electro-optical performance of different 
biphenyl-tolane isothiocyanates and 2) to formulate high birefringence mixtures with low melting temperatures. Two host mixtures were chosen as benchmarks for comparisons. The first one is our in-house high birefringence mixture UCF-1 and the second one is commercially available Merck high birefringence mixture BL038. Detailed data for both host mixtures can be found in Table 2 . There is a significant difference in the birefringence and viscosity of the host mixtures. It is especially well expressed by the FoM value which at $25^{\circ} \mathrm{C}$ was measured to be $11.6 \mu \mathrm{m}^{2} / \mathrm{s}$ and $3.1 \mu \mathrm{m}^{2} / \mathrm{s}$, respectively, for UCF-1 and BL038. This difference comes from birefringence and visco-elastic coefficient. UCF-1 has about $50 \%$ higher $\Delta \mathrm{n}$ and lower viscosity than BL038. The clearing point of UCF-1 is comparable to that of BL038. These two mixtures were doped by the eutectic compositions of compounds $1,2,3$, and 4 to form two new high birefringence mixtures, designated as ILCC-A and ILCC-B, respectively. The amount we doped was $30 \mathrm{wt} \%$ in both cases. Table 2 shows the measured physical properties of these mixtures.

The most visible impact of dopant is seen from the birefringence increase. Doping $30 \%$ of such a biphenyl-tolane increases the birefringence of the host mixtures by $12 \%$ and $29 \%$, respectively, for UCF- 1 and BL038. The larger increase in BL038 is because of its initially lower birefringence. The visco-elastic coefficient of ILCC-A and ILCC-B does not differ much from their host origins UCF-1 and BL038. In both mixtures, a small decrease was observed only near the room temperature range. Our UCF-1 host mixture contains about $26 \%$ of cyclohexyl-tolane isothiocyanates [17] which are the most viscous components therein. By doping biphenyl-tolanes we effectively reduce the percentage of the cyclohexyl containing components. As a result, the mixture's visco-elastic coefficient drops slightly. Decreased visco-elastic coefficient of ILCC-B is believed to originate from breaking symmetry of the components of BL038 which is mainly comprised of cyano compounds. The cyano biphenyls and terphenyls can pair with the neighboring compounds and form dimmers leading to a high

TABLE 2 Physical and Electro-Optical Properties of LC Mixtures

\begin{tabular}{lccccccccrc}
\hline mixture & $\begin{array}{c}\mathrm{V}_{\mathrm{th}} \\
{\left[\mathrm{V}_{\mathrm{rms}}\right]}\end{array}$ & $\varepsilon_{\|}$ & $\varepsilon_{\perp}$ & $\Delta \varepsilon$ & $\begin{array}{l}\mathrm{K}_{11} \\
{[\mathrm{pN}]}\end{array}$ & $\begin{array}{l}\mathrm{K}_{33} \\
{[\mathrm{pN}]}\end{array}$ & $\begin{array}{c}\Delta \mathrm{n} \\
633 \mathrm{~nm}\end{array}$ & $\begin{array}{c}\gamma_{1} / \mathrm{K}_{11} \\
{\left[\mathrm{~ms} / \mu \mathrm{m}^{2}\right]}\end{array}$ & $\begin{array}{c}\mathrm{FoM} \\
{\left[\mu \mathrm{m}^{2} / \mathrm{s}\right]}\end{array}$ & $\begin{array}{c}\mathrm{T}_{\mathrm{c}} \\
{\left[{ }^{\circ} \mathrm{C}\right]}\end{array}$ \\
\hline UCF-1 & 1.6 & 20.9 & 4.7 & 16.2 & 19.7 & 21.1 & 0.378 & 12.3 & 11.6 & 100 \\
BL038 & 1.6 & 19.4 & 5.0 & 14.4 & 19.1 & 22.4 & 0.257 & 30.2 & 2.2 & 100 \\
ILCC-A & 1.8 & 21.3 & 4.3 & 17.0 & 28.3 & 29.0 & 0.425 & 11.7 & 15.4 & 130 \\
ILCC-B & 1.7 & 20.1 & 4.7 & 15.4 & 22.5 & 24.5 & 0.332 & 26.3 & 4.2 & 128 \\
\hline
\end{tabular}


viscosity. We also observed a significant increase of $\mathrm{K}_{11}$ splay elastic constant in the ILCC mixtures. Finally, the FoM value of both doped mixtures is higher than that of the hosts. At room temperature, the gain is $\sim 32 \%$ and $35 \%$, respectively, for ILCC-A and ILCC-B. Similar to birefringence increase, the FoM of ILCC-B is significantly improved due to the low initial value of BL038 mixture. Further increase of FoM is possible if the same mixture is used at elevated temperatures. The improvement basically originates from the rotational viscosity

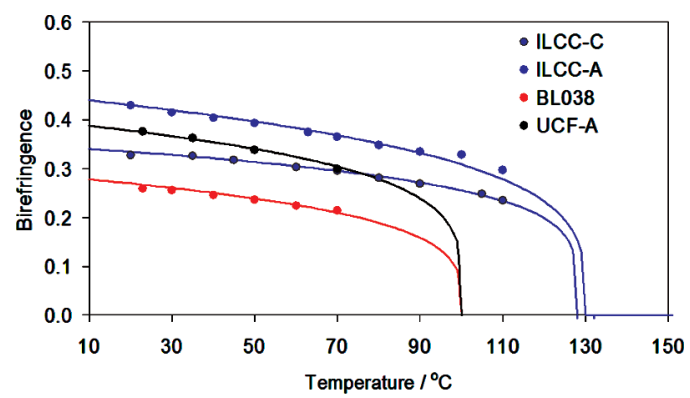

(a)

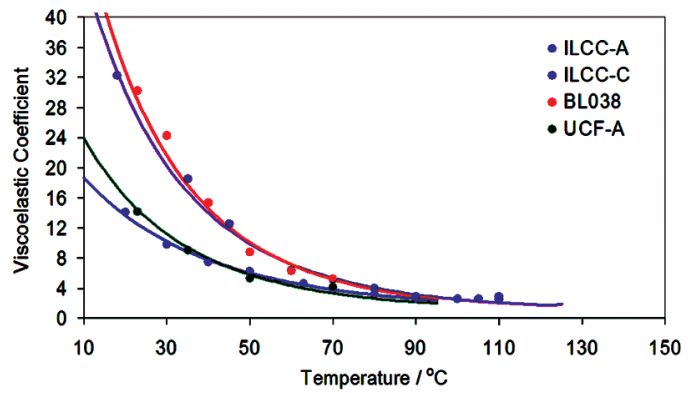

(b)

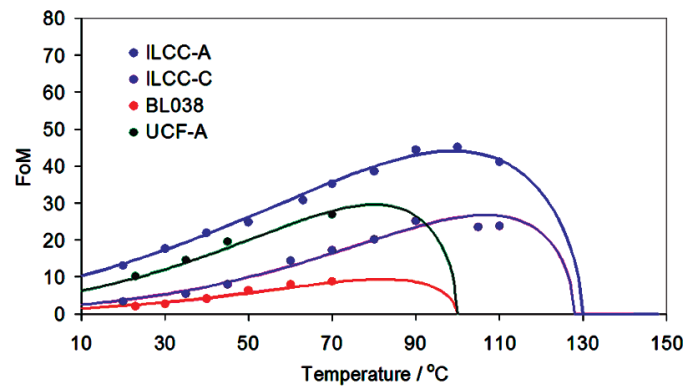

(c)

FIGURE 2 Temperature dependent (a) birefringence, (b) visco-elastic coefficient, and (c) figure-of-merit of four mixtures. 
decrease as the temperature increases. We studied birefringence, visco-elastic coefficient, and FoM of all four mixtures as a function of temperature. Detailed results are shown in Figure 2(a), 2(b) and 2(c), respectively, for birefringence, visco-elastic coefficient, and FoM. Due to the clearing temperature increase, the optimal operating temperature of the FoM of doped mixtures (the temperature at which FoM reaches its highest value) also increases. Thus, if we compare the FoM values at the optimal temperature $\left(100^{\circ} \mathrm{C}\right.$ for ILCC-A and $105^{\circ} \mathrm{C}$ for ILCC-B), the improvement is respectively $45 \%$ and $140 \%$.

\section{CONCLUSION}

We have developed some NCS-biphenyl-tolane compounds and mixtures and evaluated their physical properties. The fluoro substitutions lower the melting temperatures, but also increase the viscosity. A certain compromise needs to be taken between high FoM and melting temperature of the compounds and mixtures. These high FoM mixtures are especially attractive for long wavelength applications, such as laser beam steering and spatial light modulators [18]. The UV stability is not a big concern for IR applications.

\section{REFERENCES}

[1] McManamon, P. F., Dorschner, T. A., Corkum, D. L., Friedman, L., Hobbs, D. S., Holz, M., Liberman, S., Nguyen, H. Q., Resler, D. P., Sharp, R. C., \& Watson, E. A. (1996). Proc. IEEE, 84, 268.

[2] Lu, Y., Du, F., Lin, Y. H., \& Wu, S. T. (2004). Opt. Express, 12, 1221.

[3] Spadło, A., Dąbrowski, R., Filipowicz, M., Stolarz, Z., Przedmojski, J., Gauza, S., Fan, Y. H., \& Wu, S. T. (2003). Liq. Cryst., 30, 191.

[4] Wu, S. T., Margerum, J. D., Meng, B., Dalton, L. R., Hsu, C. S., \& Lung, S. H. (1992). Appl. Phys. Lett., 61, 630.

[5] Wu, S. T., Neubert, M., Keast, S. S., Abdallah, D. G., Lee, S. N., Walsh, M. E., \& Dorschner, T. A. (2000). Appl. Phys. Lett., 77, 957.

[6] Wu, S. T., Hsu, C. S., \& Shyu, K. F. (1999). Appl. Phys. Lett., 74, 344.

[7] Wu, S. T., Hsu, C. S., Chuang, Y. Y., \& Cheng, H. B. (2000). Jpn. J. Appl. Phys. Part 2, 39, L38.

[8] Seed, A. J., Toyne, K. J., Goodby, J. W., \& Hird, M. (2000). J. Mater. Chem., 10, 2069.

[9] Sekine, C., Konya, N., Minai, M., \& Fujisawa, K. (2001). Liq. Cryst., 28, 1361.

[10] Sekine, C., Ishitobi, M., Iwakura, K., Minai, M., \& Fujisawa, K. (2002). Liq. Cryst., $29,355$.

[11] Sekine, C., Konya, N., Minai, M., \& Fujisawa, K. (2001). Liq. Cryst., 28, 1495.

[12] Catanescu, O. \& Chien, L. C. (2006). Liq. Cryst., 33, 115.

[13] Gauza, S., Wu, S. T., Spadlo, A., \& Dabrowski, R. (2006). J. Display Technology, $2,247$.

[14] Gauza, S., Wen, C. H., Wu, B., Wu, S. T., Spadlo, A., \& Dabrowski, R. (2006). Liq. Cryst., 33, 705. 
[15] Lin, P. T., Wu, S. T., Chang, C. Y., \& Hsu, C. S. (2004). Mol. Cryst. Liq. Cryst., $411,243$.

[16] Wu, S. T., Efron, U., \& Hess, L. D. (1984). Appl. Opt., 23, 3911.

[17] Gauza, S., Wang, H., Wen, C. H., Wu, S. T., Seed, A. J., \& Dabrowski, R. (2003). Jpn. J. Applied Physics Part 1, 42, 3463.

[18] Harriman, J., Gauza, S., Wu, S. T., Wick, D., Bagwell, B., Martinez, T., Payne, D., \& Serati, S. (2006). Proc. SPIE, 6135, 1. 